\title{
Optimal Placement in a Limit Order Book
}

\author{
Xin Guo * Adrien de Larrard ${ }^{\dagger}$ Zhao Ruan ${ }^{\ddagger}$
}

August 14, 2013

\begin{abstract}
This paper proposes and studies an optimal placement problem in a limit order book. Two simple models are proposed: one with price impact and one without price impact. For the first model, optimal placement strategies for both single-period and multi-period cases are derived. For the second model, it is shown that the optimal strategy never mixes market orders and (best) limit orders; in particular, with special choices of parameters, the optimal strategy reduces to the VWAP type of Bertsimas and Lo (1998) for the optimal execution problem.
\end{abstract}

\section{Introduction}

Technological innovation has completely transformed the fundamentals of the financial market. As a result, automatic and electronic order-driven trading platforms have largely replaced the traditional floor-based trading. In an electronic order-driven market, orders arrive at the exchange and wait in the Limit Order Book ( $L O B)$ to be executed. In US, high-frequency trading firms represent $2 \%$ of the approximately 20,000 firms operating today, but account for $73 \%$ of all equity orders volume. In most exchanges, order flow is heavy with thousands of orders in seconds and tens of thousands of price changes in a day for a liquid stock. Meanwhile, the time for the execution of a market order has dropped below one millisecond. This new era of trading is commonly referred to as High Frequency Trading (HFT) or Algorithmic Trading.

Limit order book (LOB). In an order-driven market, there are two types of buy/sell orders for market participants to post: market orders and limit orders. A limit order is an order to trade a certain amount of security (stocks, futures, etc.) at a given specified price. The lowest price for which there is an outstanding limit sell order is called the best ask price and the highest limit buy price is called the best bid price. Limit orders are collected and posted in the LOB, which contains the quantities and the price at each price level for all limit buy and sell orders. A market order is an order to buy/sell a certain amount of the equity at the best available price in the LOB. It is then matched with the best available price and a trade occurs immediately and the LOB is updated accordingly. A limit order stays in the LOB until it is executed against a market order or until it is canceled; cancellation is allowed at

*Dept of Industrial Engineering and Operations Research, UC at Berkeley, Berkeley, CA 94720-1777. Email: xinguo@ieor.berkeley.edu. Tel: 1-510-642-3615.

†175, rue du Chevaleret, Laboratoire de Probabilités et Modèles Aléatoires, 75013, Paris, France. Email: larrard@clipper.ens.fr.

${ }^{\ddagger}$ Dept of Industrial Engineering and Operations Research, UC at Berkeley, Berkeley, CA 94720-1777. Email: rzdream@gmail.com. 
any time. In essence, the closer a limit order is to the best bid/ask, the faster it may be executed. Most exchanges are based on First-In-First-Out (FIFO) policy for orders on the same price level, although some derivatives on some exchanges have the pro-rate microstructure. That is, an incoming market order is dispatched on all active limit orders at the best price, with each limit order contributing to execution in proportion to its volume. In this paper, unless otherwise specified, we always focus the discussions on the FIFO market, with extensions to the pro-rate microstructure whenever appropriate.

Order books are available with different levels of details. For example, the so called "level-1 order book" contains the best price level of the order book, while "level-2 order book" provides the prices and quantities of the best five levels on both the ask and the bid sides. The following table is a typical example showing the dynamics of the limit order book of the top 5 levels: a market sell order of size 1200, followed by a limit ask order of size 400 at price 9.08, and then a cancellation of size 23 for limit ask order ar the price 9.10 .

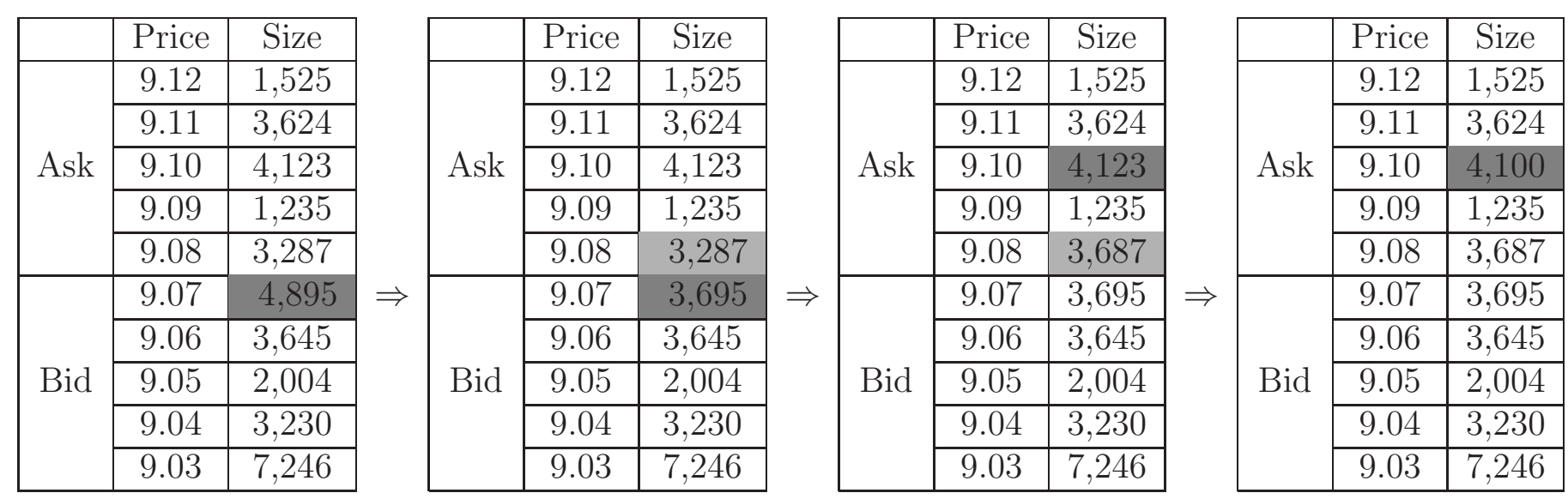

Table 1: A market sell order with size of 1200, a limit ask order with size of 400 at 9.08, and a cancelation of 23 shares of limit ask order at 9.10, in sequence.

The problem of optimal placement. Optimal placement is the most basic element in the design of algorithm trading. It studies how to optimally place the small-sized orders in the LOB. Specifically, when using limit orders, traders do not need to pay the spread and most of the time even get a rebate (say, $r$ ). This rebate structure varies from exchange to exchange and leads to different optimization problems. For instance, in HK stock exchange, successful executions of limit orders get a discount (i.e., a fixed percentage of the execution price) whereas in other places such as the London stock exchange, the discount may be a fixed amount. This rebate, however, comes with an execution risk as there is no guarantee of execution for limit orders. On the other hand, when using market orders, one has to pay both the spread between the limit and the market orders and the fee $f$ in exchange for a guaranteed immediate execution. Thus, traders must decide: given a number of shares to buy or sell, should one use market orders, or limit orders, or both? How many orders to be placed at different price levels? What is the optimal sequence of order placement in a give time frame with multi-trades? In essence, traders have to balance the tradeoff between paying the spread and fees vs. execution/inventory risk when placing market orders and limit orders.

In essence, the problem of optimal placement is as follows:

- One needs to buy $N$ orders by time $T>0$ ( $\mathrm{T} \approx 1 / 5$ minutes); 
- One can split the $N$ orders into $\left(N_{0, t}, N_{1, t}, \ldots\right)$, where $N_{0, t} \geq 0$ is the number of market orders at time $t=0,1, \cdots, T, N_{1, t}$ is the number of orders at the best bid at time $t, N_{2, t}$ is the number of orders at the second best bid at time $t$, and so on;

- No intermediate selling is allowed before time $T$;

- If the limit orders are not executed by time $T$, one has to buy the non-executed orders at the market price at time $T$;

- When one share of limit order is executed, the market gives a rebate of $r>0$;

- When a share of market order is submitted, there is a fee of $f>0$;

- Given $N$ and $T$, the goal is to find the optimal strategy $\left(N_{0, t}, N_{1, t}, \ldots, N_{k, t}\right)_{t=0,1, \cdots, T}$ to minimize the overall total expected cost.

Clearly the answer to this problem depends on the expected costs at each level of LOB where an order is placed and in particular, the probability of execution at each level by the end of the period for each order.

There is also a price impact issue in the optimal placement problem. The major modeling hypothesis on price impact is that any trading strategy, especially that involves a large amount of buying and selling within a short period of time, will have an impact on the stock price: too many large orders may depress the price and reduce the potential profit whereas too many small transactions may be costly and may take too long to complete. The empirical study by Cont, Kukanov, and Stoikov (2013) [19] verifies the existence of price impact at the trade level and suggests that the price impact is more or less linear.

Optimal placement problem is closely related to but different from the well-known optimal execution problem, in which price impact is the main modeling factor. Recall that the optimal execution problem is to slice big orders into smaller ones on a daily/weekly basis in order to minimize the price impact or to maximize some expected utility function; see for instance, Bertsima and Lo (1998) [10], Almgren and Chriss (1999, 2000) [5, 6], Almgren (2003) [4, Almgren and Lorenz(2007) [7, Schied and Schöneborn (2009) [47, Weiss (2009) [50], Alfonsi, Fruth, and Schied (2010) [1], Gatheral (2010) [24, Predoiu, Shaiket, and Shreve (2010) [43], Schied, Schöneborn, and Tehranchi (2010) 48, and Alfonsi, Schied, and Slynko (2012)[2], Gatheral and Schied (2011)[25], Forsyth, Kennedy, Tse, and Windcliff (2011) 23], and Guo and Zervos (2012) 30], and Obizhaeva and Wang (2013) [42]. Optimal placement problem, on the other hand, is on a smaller (10-100 seconds) time scale and mostly for different type of (i.e., HFT) traders (see Kirilenko et. al. (2011) [36]).

To our best knowledge, there is essentially no existing result on the optimal placement problem except for Hult and Kiessling (2010) [35], who consider a special version of the problem with $N=1$ using a high-dimensional Markov chain model for the state and evolution of the entire LOB. Using the potential theory for Markov chains, they derive conditions for the existence of an optimal strategy and provide a value-iteration algorithm to numerically find the optimal strategy.

Our results. In this paper, we will analyze the optimal placement problem in two steps.

- First, we will propose a correlated random walk model without the price impact factor. In this model, we will analyze the optimal strategy in a single period where orders can only be placed at the beginning and at the end time $T$; we will show that the optimal strategy will involve only the best bid and market orders. Based on this analysis, we move on to a multi-period model where one is allowed to adjust and cancel any non-executed (limit) orders after each price movement/each time 
period. Here we assume the choices are market order, the best bid order, or no order placement at any time $t<T$. We will show that the optimal strategy at each time $t$ is a threshold type.

- Then we will propose a model taking into account the price impact. We will propose a martingale price model with an additive price impact and assume that the choices are between the best bid and the market orders. In this case, we will analyze the structure of the optimal strategy and show that the optimal trading strategy never mixes the limit and market orders. As a result, the optimal strategy can be computed recursively. In a special and degenerate case of the model, our result reduces to the VWAP strategy in the sense of Bertsimas and Lo (1998) [10] for the optimal execution problem.

\section{Models and Analysis}

\subsection{Correlated Random Walk Model and Analysis}

The model. We will first propose and analyze a correlated random walk model for the dynamics of the bid/ask price. In this model, we will assume that

1. The spread between the best bid price and the best ask price is always 1 tick;

2. The best ask price increases or decrease 1 tick at each time step $t=0,1, \cdots, T$;

3. There is no price impact.

Moreover, let $A_{t}$ be the best ask price at time $t$, then

$$
A_{t}=\sum_{i=1}^{t} X_{i}, A_{0}=0
$$

with $\left(X_{i}\right)_{i \geq 0}$ taking values 1 and $-1,\left(X_{i}, X_{i+1}\right)$ a two-dimensional Markov chain with $\mathbb{P}\left(X_{1}=1\right)=\bar{p}=$ $1-\mathbb{P}\left(X_{1}=-1\right)$ for some $\bar{p} \in[0,1]$, and

$$
\mathbb{P}\left(X_{i+1}=1 \mid X_{i}=1\right)=\mathbb{P}\left(X_{i+1}=-1 \mid X_{i}=-1\right)=p<\frac{1}{2}, \text { for } i=0,1, \cdots, T-1 .
$$

Note that this model is a simple case of the well-known correlated random walk model. (See Goldstein (1951) [27], Mohan(1955) [39], Gillis (1955) [26], and Renshaw and Henderson (1981) [40].) The particular choice of $p<\frac{1}{2}$ makes the price "mean revert," as consistent with some observation in high-frequency tradings. (See for instance Chen and Hall (2013) [16] for some statistical analysis on this.)

To analyze this model for the optimal placement problem, first note that without price impact assumption it suffices to consider $N=1$. Next, critical to our analysis is $A_{t}$ and $Y_{t}$, where

$$
Y_{t}=\min _{1 \leq i \leq t} A_{i}
$$

Probability distribution of $A_{t}$ and $Y_{t}$. For ease of exposition, let us call any price change of $A_{t}$ from increase to decrease (or decrease to increase) as a direction change, and suppose there are $i$ such direction changes between from 0 to $T, 0 \leq i \leq T$. Then it is not difficult to see that

$$
\mathbb{P}\left(A_{T}=k \mid \text { number of direction changes is } \mathrm{i}\right)=\left\{\begin{array}{lr}
p^{T-i-1}(1-p)^{i} L_{T, k}^{i}, & \frac{T+k}{2} \in \mathbb{N} \&|k| \leq T \\
0, & \text { otherwise. }
\end{array}\right.
$$


Where

$$
L_{T, k}^{i}=\left[(1-\bar{p})\left(\begin{array}{c}
\frac{T+k}{2}-1 \\
\left\lfloor\frac{i+1}{2}\right\rfloor-1
\end{array}\right)\left(\begin{array}{c}
\frac{T-k}{2}-1 \\
\left\lfloor\frac{i+2}{2}\right\rfloor-1
\end{array}\right)+\bar{p}\left(\begin{array}{c}
\frac{T+k}{2}-1 \\
\left\lfloor\frac{i+2}{2}\right\rfloor-1
\end{array}\right)\left(\begin{array}{c}
\frac{T-k}{2}-1 \\
\left\lfloor\frac{i+1}{2}\right\rfloor-1
\end{array}\right)\right] .
$$

Summing up over all possible $i$ we

$$
\mathbb{P}\left(A_{T}=k\right)=\left\{\begin{array}{lr}
\sum_{i=1}^{T-k} p^{T-i-1}(1-p)^{i} L_{T, k}^{i}, & \frac{T+k}{2} \in \mathbb{N} \&|k| \leq T \\
0, & \text { otherwise. }
\end{array}\right.
$$

Lemma 1. If $\frac{T-k}{2} \in \mathbb{N}$ and $k>0$, then $\mathbb{P}\left(Y_{T}=-k\right)=\mathbb{P}\left(A_{T}=-k\right)$.

Proof. Notice that

$$
\begin{aligned}
\mathbb{P}\left(Y_{T} \leq-k\right) & =\mathbb{P}\left(Y_{T} \leq-k, A_{T} \leq-k\right)+\mathbb{P}\left(Y_{T} \leq-k, A_{T}>-k\right) \\
& =\mathbb{P}\left(A_{T} \leq-k\right)+\mathbb{P}\left(Y_{T} \leq-k, A_{T}>-k\right)
\end{aligned}
$$

and

$$
\begin{aligned}
\mathbb{P}\left(Y_{T}=-k\right) & =\mathbb{P}\left(Y_{T} \leq-k\right)-\mathbb{P}\left(Y_{T} \leq-k-1\right) \\
& =\mathbb{P}\left(A_{T}=-k\right)+\mathbb{P}\left(Y_{T} \leq-k, A_{T} \geq-k+1\right)-\mathbb{P}\left(Y_{T} \leq-k-1, A_{T} \geq-k\right) .
\end{aligned}
$$

Since $\frac{T-k}{2} \in \mathbb{N},\left\{A_{T} \geq-k+1\right\}=\left\{A_{T} \geq-k+2\right\}$. Then it is sufficient to show

$$
\mathbb{P}\left(Y_{T} \leq-k, A_{T} \geq-k+2\right)=\mathbb{P}\left(Y_{T} \leq-k-1, A_{T} \geq-k\right)
$$

For $\forall \omega \in\left\{Y_{T} \leq-k-1, A_{T} \geq-k\right\}$, denote $X(\omega)=\left(X_{1}^{\omega}, X_{2}^{\omega}, \cdots, X_{n}^{\omega}\right)$ and $A(\omega)=\left(A_{1}^{\omega}, A_{2}^{\omega}, \cdots, A_{T}^{\omega}\right)$. Let $\tau(\omega)=\sup \left\{i: A_{i}^{\omega}=Y_{T}(\omega)\right\}$, which is the last time that $A(\omega)$ reaches the level of $Y_{T}(\omega)$. Then define $h:\left\{Y_{T} \leq-k, A_{T} \geq-k+2\right\} \rightarrow\left\{Y_{T} \leq-k-1, A_{T} \geq-k\right\}$ as $h(\omega)=\tilde{\omega}$, where $\tilde{\omega}$ satisfies

$$
X_{i}^{\tilde{\omega}}=\left\{\begin{array}{cl}
X_{i}^{\omega}, & i \neq \tau(\omega)+1, \\
-1, & i=\tau(\omega)+1 .
\end{array}\right.
$$

First, we show that $h$ is well defined, i.e., $\tilde{\omega} \in\left\{Y_{T} \leq-k-1, A_{T} \geq-k\right\}$, and that this mapping keeps the number of direction changes. Since $\tau(\omega)$ is the last time $A(\omega)$ reaches the level of $Y_{T}(\omega)$ and $A_{T}(\omega)-Y_{T}(\omega) \geq 2$, we have $X_{\tau(\omega)}^{\omega}=-1$ and $X_{\tau(\omega)+1}^{\omega}=X_{\tau(\omega)+2}^{\omega}=1$. Since $\tilde{\omega}$ only changes the step $\tau(\omega)+1$ from 1 to -1 , we know that $A_{\tau(\omega)+1}^{h(\omega)} \leq-k-1$ and $Y_{T}(h(\omega))=Y_{T}(\omega)-2 \geq-k$. Thus $\tilde{\omega} \in\left\{Y_{T} \leq-k-1, A_{T} \geq-k\right\}$. And we also noted that $h$ keeps the number of direction changes from $\omega$ to $\tilde{\omega}$. Since $\tau(\omega) \geq 1$ for all $k>0$, we have $X_{1}^{\omega}=X_{1}^{h(\omega)}$ and

$$
\mathbb{P}(\omega)=\mathbb{P}(\tilde{\omega}) .
$$

Second, we show that $h$ is an injection. Suppose $\omega_{1}, \omega_{2} \in\left\{Y_{n} \leq-k, A_{n} \geq-k+2\right\}$ satisfying $h\left(\omega_{1}\right)=h\left(\omega_{2}\right)$. If $\tau\left(\omega_{1}\right)=\tau\left(\omega_{2}\right)$, then obviously we have $\omega_{1}=\omega_{2}$. And if $\tau\left(\omega_{1}\right) \neq \tau\left(\omega_{2}\right)$, and W.O.L.G. $\tau\left(\omega_{1}\right)<\tau\left(\omega_{2}\right)$. Then for any $i \leq \tau\left(\omega_{1}\right)$ and $i>\tau\left(\omega_{2}\right)$, we have $X_{i}^{\omega_{1}}=X_{i}^{\omega_{2}}$. For $\tau\left(\omega_{1}\right)+1$, we have $X_{\tau\left(\omega_{1}\right)+1}^{\omega_{2}}=-1$ and $X_{\tau\left(\omega_{1}\right)+1}^{\omega_{2}}=1$. Thus $A_{\tau\left(\omega_{1}\right)+1}^{\omega_{2}}=A_{\tau\left(\omega_{1}\right)}^{\omega_{1}}-1=Y_{T}\left(\omega_{1}\right)-1$. Then

$$
Y_{T}\left(\omega_{2}\right) \leq A_{\tau\left(\omega_{1}\right)+1}^{\omega_{2}} \leq Y_{T}\left(\omega_{1}\right)-1
$$


And since $A_{\tau\left(\omega_{2}\right)+1}^{\omega_{1}}=A_{\tau\left(\omega_{2}\right)+1}^{\omega_{2}}=Y_{T}\left(\omega_{2}\right)+1$, we have $A_{\tau\left(\omega_{2}\right)+1}^{\omega_{1}} \leq Y_{T}\left(\omega_{1}\right)$, which is contradictory to the definition of $\tau\left(\omega_{1}\right)$. Thus $\tau\left(\omega_{1}\right)=\tau\left(\omega_{2}\right)$ and $\omega_{1}=\omega_{2}$. That is, $h$ is an injection.

Third, we show that $h$ is a surjection, i.e., for any $\tilde{\omega} \in\left\{Y_{T} \leq-k-1, A_{T} \geq-k\right\}$, there exists $\omega \in\left\{Y_{T} \leq-k, A_{T} \geq-k+2\right\}$, such that $h(\omega)=\tilde{\omega}$. Let $\tilde{\tau}(\tilde{\omega})=\inf \left\{i: A_{i}^{\omega}=Y_{n}(\omega)\right\}$, i.e. the first time $A(\tilde{\omega})$ reaches $Y_{T}(\tilde{\omega})$. Then define $\omega$ by

$$
X_{i}^{\omega}= \begin{cases}X_{i}^{\tilde{\omega}}, & i \neq \tilde{\tau}(\tilde{\omega}), \\ 1, & i=\tilde{\tau}(\tilde{\omega}) .\end{cases}
$$

Since $\tilde{\tau}(\tilde{\omega})$ is the first time that $A(\tilde{\omega})$ reaches $Y_{T}(\omega)$, by definition $A_{i}^{\omega} \geq Y_{T}(\omega)+1$ for any $i<\tilde{\tau}(\tilde{\omega})$, and $A_{i}^{\omega} \geq A_{i}^{\tilde{\omega}}+2 \geq Y_{T}(\omega)+2$ for any $i \geq \tilde{\tau}(\tilde{\omega})$, Thus $\tilde{\tau}(\tilde{\omega})-1$ is the last time that $A(\omega)$ reaches its lowest position. Then by the definition of $h, h(\omega)=\tilde{\omega}$. Hence $h$ is a surjection. Then follows that $h$ is indeed a bijection from $\left\{Y_{T} \leq-k, A_{T} \geq-k+2\right\}$ to $\left\{Y_{T} \leq-k-1, A_{T} \geq-k\right\}$. In addition, the equality (2.4) suggests

$$
\mathbb{P}\left\{Y_{T} \leq-k-1, A_{T} \geq k\right\}=\mathbb{P}\left\{Y_{T} \leq-k-1, A_{T} \geq-k\right\}
$$

and

$$
\mathbb{P}\left(Y_{T}=-k\right)=\mathbb{P}\left(A_{T}=-k\right)
$$

Remark 1. Note that with the degenerate case $p=1 / 2$, the correlated random walk model becomes a simple random walk, and the reflection principle argument leads to the same result (see P.97 to P.100 in Redner and Sidney (2001) [41]).

Next, we have the monotone property of the distribution of $Y_{t}$.

Proposition 2. $\mathbb{P}\left(Y_{T}=-k\right)$ is a decreasing function with respect to $k, k=1,2, \ldots, T$.

Proof. For $\forall \omega \in\left\{Y_{T}=-k-1\right\}$, let $\tau(\omega)=\inf \left\{i: A_{i}^{\omega}=Y_{T}^{\omega}=\right\}$, i.e. the first time that $A^{\omega}$ reaches the lowest position. Since $k>0$, thus $\tau(\omega)$ exists (otherwise the process will never reach -2 ). Define $g$ on $\left\{Y_{T}=-k-1\right\}$ as $g(\omega)=\tilde{\omega}$, where $\tilde{\omega}$ satisfies

$$
X(\tilde{\omega})= \begin{cases}X_{i}^{\omega}, & i \neq \tau(\omega), \\ 1, & i=\tau(\omega) .\end{cases}
$$

That is, $g$ changes $X_{\tau(\omega)}$ from a "downward" to an "upward". Since $\tau(\omega)$ is the first time that $A^{\omega}$ hit the lowest position, then it is clear that $Y_{T}(\tilde{\omega})=-k$. Thus $g(\omega) \in\left\{Y_{T}=-k\right\}$. For $\forall \omega_{1}$ and $\omega_{2} \in$ $\left\{Y_{T}=-k-1\right\}$, if $g\left(\omega_{1}\right)=g\left(\omega_{2}\right)=\tilde{\omega}$, then let $\tau_{1}=\tau\left(\omega_{1}\right)$ and $\tau_{2}=\tau\left(\omega_{2}\right)$. If $\tau_{1}=\tau_{2}$, then it is easy to see that for any $i<\tau_{1}, X_{i}^{\omega_{1}}=X_{i}^{\omega_{2}}$. Similarly for $i=\tau_{1}$ and $i>\tau_{1}$. Thus $\omega_{1}=\omega_{2}$. If $\tau_{1} \neq \tau_{2}$ and W.O.L.G. $\tau_{1}<\tau_{2}$. Then for any $i<\tau_{1}, X_{i}^{\omega_{1}}=X_{i}^{\omega_{2}} ; A_{i}^{\omega_{1}}=A_{i}^{\omega_{2}}-2$ for $\tau_{1} \leq i<\tau_{2}$; and $X_{i}^{\omega_{2}}=-1$ and $X_{i}^{\omega_{1}}=1$ for $i=\tau_{2}$. Thus $A_{\tau_{2}}^{\omega_{2}}=A_{\tau_{1}}^{\omega_{2}} \geq Y_{T}\left(\omega_{1}\right)+1=-k$, which is contradictory to the definition of $\omega_{2}$. Hence $\tau_{1}=\tau_{2}$ and $\omega_{1}=\omega_{2}$. Thus $g$ is an injection. Note that $g(\omega)$ has at least the same number of changes of directions, $\mathbb{P}(g(\omega)) \geq \mathbb{P}(\omega)$. Thus $\mathbb{P}\left\{Y_{T}=-k-1\right\} \leq \mathbb{P}\left\{Y_{T}=-k\right\}$. Similarly, $\mathbb{P}\left\{Y_{T}=-k\right\} \leq \mathbb{P}\left\{Y_{T}=-k+1\right\}$. 


\subsubsection{Analysis of Optimal Placement Strategy: the Single-period Case}

With these preliminary analysis, we will first consider the optimal placement in a single period, by which we mean that

- One only places her order at the beginning, i.e. $t=0$;

- If the order is placed as a limit order and the limit bid order is not executed before time $T$, then one has to use market orders to buy the stock at time $T$;

- A limit order placed at price $-k$ will be executed with probability 1 if $Y_{T} \leq-k$ and with probability $q$ if $Y_{T}=-k+1$. Otherwise, a market order has to be used at time $T$ to replace the existing non-executed order.

Comparing expected costs at each level of LOB. Since $N=1$, it amounts to compare the expected cost of placing the order at each level of the LOB.

Recall that there is a fee $f$ for each market order and a rebate $r$ for an executed limit order. Let

$C_{0}^{q}$ denote the expect cost of a market order and $C_{i}^{q}$ the expected cost of a limited order placed at the price $i$ ticks lower than the initial best ask price. Although the costs are functions of $f, r, T, p, q, \bar{p}$, for ease of exposition we use the superscript $q$ to highlight their dependence on $q$.

Obviously, $C_{0}^{q}=f$, and

$$
\begin{aligned}
C_{k}^{q}= & \mathbb{P}\left(Y_{T} \leq-k\right)(-k-r)+q \mathbb{P}\left(Y_{T}=-k+1\right)(-k-r) \\
& +\mathbb{P}\left(Y_{T}>-k+1\right)\left(\mathbb{E}\left[A_{T} \mid Y_{T}>-k+1\right]\right)+(1-q) \mathbb{P}\left(Y_{T}=-k+1\right)\left(\mathbb{E}\left[A_{T} \mid Y_{T}=-k+1\right]\right) .
\end{aligned}
$$

Lemma 3. For $k=1,2, \ldots T-2$,

$$
\mathbb{P}\left(Y_{T}=-k\right)\left(k+\mathbb{E}\left[A_{T} \mid Y_{T}=-k\right]\right)-\mathbb{P}\left(Y_{T}=-k-1\right)\left(k+2+\mathbb{E}\left[A_{T} \mid Y_{T}=-k-1\right]\right) \geq 0 .
$$

Proof. Define the same mapping $g$ from $\left\{Y_{T}=-k-1\right\}$ to $\left\{Y_{T}=-k\right\}$ as in the proof of Proposition 2. Now $g$ is an injection and $\mathbb{P}(\omega) \leq \mathbb{P}(g(\omega))$ for any $\omega \in\left\{Y_{T}=-k-1\right\}$, because $g$ will not decrease the number of direction changes of the path from $\omega$ to $g(\omega)$. Moreover, because $g$ changes one "downward" edge to one "upward" edge, we have $A_{T}(g(\omega))=A_{T}(\omega)+2$. Thus

$$
\begin{aligned}
& \mathbb{P}\left(Y_{T}=-k-1\right)\left(k+2+\mathbb{E}\left[A_{T} \mid Y_{T}=-k-1\right]\right) \\
= & \sum_{\omega \in\left\{Y_{T}=-k-1\right\}} \mathbb{P}(\omega)\left(k+2+A_{T}(\omega)\right) \\
\leq & \sum_{g(\omega) \in g\left(\left\{Y_{T}=-k-1\right\}\right)} \mathbb{P}(g(\omega))\left(k+A_{T}(g(\omega))\right) \\
\leq & \sum_{g(\omega) \in\left\{Y_{T}=-k\right\}} \mathbb{P}(g(\omega))\left(k+A_{T}(g(\omega))\right) .
\end{aligned}
$$

The second to the last inequality holds because $g$ is an injection, and the last inequality holds because $A_{T}(g(\omega))+k \geq 0$ for $\forall g(\omega) \in\left\{Y_{T}=-k\right\}$.

Proposition 4. Given $r$, $f, T, p$, and $\bar{p}, C_{1}^{q}<C_{2}^{q}<\ldots<C_{T}^{q}<C_{T+1}^{q}$. 
Proof. Note that

$$
C_{k}^{q}=q C_{k}^{1}+(1-q) C_{k}^{0}
$$

Then, it suffices to show

$$
\begin{aligned}
& C_{1}^{0}<C_{2}^{0}<\ldots<C_{T}^{0}<C_{T+1}^{0}, \\
& C_{1}^{1}<C_{2}^{1}<\ldots<C_{T}^{1}<C_{T+1}^{1} .
\end{aligned}
$$

Let $b_{k}^{0}=C_{k+1}^{0}-C_{k}^{0}$ and $b_{k}^{1}=C_{k+1}^{1}-C_{k}^{1}$ for $k=1,2, \ldots, T$. We first show that $b_{k}^{0}>0$ for $k=1,2, \ldots, T$,

$$
b_{k}^{0}=-\mathbb{P}\left(Y_{T}<-k\right)+(r+f+k) \cdot \mathbb{P}\left(Y_{T}=-k\right)+\mathbb{P}\left(Y_{T}=-k\right) \mathbb{E}\left[A_{T} \mid Y_{T}=-k\right] .
$$

Since $r, f \geq 0$, it is enough to show that for $k=1,2, \ldots, T$,

$$
\tilde{b}_{k}^{0}=-\mathbb{P}\left(Y_{T}<-k\right)+k \mathbb{P}\left(Y_{T}=-k\right)+\mathbb{P}\left(Y_{T}=-k\right) \mathbb{E}\left[A_{T} \mid Y_{T}=-k\right]>0
$$

First,

$$
\begin{aligned}
b_{T}^{0} & =f-\left(\mathbb{E}\left\{A_{T} \mid Y_{T}>-T\right\}+f\right) \mathbb{P}\left\{Y_{T}>-T\right\}-\mathbb{P}\left\{Y_{T}=-T\right\}(-T-r) \\
& =f+\mathbb{E}\left\{A_{T} \mid Y_{T}=-T\right\} \mathbb{P}\left\{Y_{T}=-T\right\}-f \mathbb{P}\left\{Y_{T}>-T\right\}-\mathbb{P}\left\{Y_{T}=-T\right\}(-T-r) \\
& =f-T \mathbb{P}\left\{Y_{T}=-T\right\}-f \mathbb{P}\left\{Y_{T}>-T\right\}-\mathbb{P}\left\{Y_{T}=-T\right\}(-T-r) \\
& =f-f+(f+r) \mathbb{P}\left\{Y_{T}=-T\right\} \\
& \geq 0 .
\end{aligned}
$$

Secondly, note $\mathbb{E}\left[A_{T} \mid Y_{T}=-T+1\right]=-T+2$, and by Proposition 2, we have

$$
\begin{aligned}
\tilde{b}_{T-1}^{0} & =-\mathbb{P}\left(Y_{T}=-T\right)+(T-1) \mathbb{P}\left(Y_{T}=-T+1\right)+\mathbb{P}\left(Y_{T}=-T+1\right) \mathbb{E}\left[A_{T} \mid Y_{T}=-T+1\right] \\
& =-\mathbb{P}\left(Y_{T}=-T\right)+\mathbb{P}\left(Y_{T}=-T+1\right) \\
& >0
\end{aligned}
$$

And for $k=1,2, \ldots, T-2$,

$$
\begin{aligned}
\tilde{b}_{k}^{0}-\tilde{b}_{k+1}^{0} & =-\mathbb{P}\left(Y_{T}=-k-1\right)+k \mathbb{P}\left(Y_{T}=-k\right)+\mathbb{E}\left[A_{T} \mid Y_{T}=-k\right] \mathbb{P}\left(Y_{T}=-k\right) \\
& -(k+1) \mathbb{P}\left(Y_{T}=-k-1\right)-\mathbb{E}\left[A_{T} \mid Y_{T}=-k-1\right] \mathbb{P}\left(Y_{T}=-k-1\right) \\
& =\mathbb{P}\left(Y_{T}=-k\right)\left(k+\mathbb{E}\left[A_{T} \mid Y_{T}=-k\right]\right)-\mathbb{P}\left(Y_{T}=-k-1\right)\left(k+2+\mathbb{E}\left[A_{T} \mid Y_{T}=-k-1\right]\right) \geq 0 .
\end{aligned}
$$

The last inequality is from lemma 3. Thus recursively we see that $\tilde{b}_{k}^{0}, i=1,2, \ldots, T-1$ are positive, which means inequality (2.7) holds. Then for $q=1$,

$$
\begin{aligned}
C_{k}^{1} & =(-k-r) \mathbb{P}\left(Y_{T} \leq-k+1\right)+\mathbb{P}\left(Y_{T} \geq-k+2\right) \mathbb{E}\left[A_{T} \mid Y_{T} \geq-k+2\right] \\
& =C_{k-1}^{0}-\mathbb{P}\left(Y_{T} \leq-k+1\right) .
\end{aligned}
$$

Since $C_{k}^{0}$ is increasing and $\mathbb{P}\left(Y_{T}\right) \leq-k+1$ as $k$ increases, the inequality (2.8) is clear. 
Comparison of $C_{0}^{q}$ and $C_{1}^{q}$ Based on the above analysis, we see that to derive the optimal order place strategy, it suffices to compare $C_{0}^{q}$ and $C_{1}^{q}$.

First, we have,

Proposition 5. In a single period model with $\bar{p}=\frac{1}{2}$, the optimal strategy is to peg the bid. That is, it is optimal to always place the order at the best bid price.

Proof. This is obvious: as $\mathbb{P}\left\{Y_{T}=-T-1\right\}=0, C_{T+1}^{0}=f+\mathbb{E}\left(A_{T}\right)=f$, and

$$
\begin{aligned}
C_{T+1}^{1} & =C_{T}^{0}-\mathbb{P}\left(Y_{T} \leq-T\right) \\
& =\left(\mathbb{E}\left\{A_{T} \mid Y_{T}>-T\right\}+f\right) \mathbb{P}\left\{Y_{T}>-T\right\}+\mathbb{P}\left\{Y_{T}=-T\right\}(-T-r-1) \\
& =-\mathbb{E}\left\{A_{T} \mid Y_{T}=-T\right\} \mathbb{P}\left\{Y_{T}=-T\right\}+f \mathbb{P}\left\{Y_{T}>=-T\right\}+\mathbb{P}\left\{Y_{T}=-T\right\}(-T-r-1) \\
& =T \mathbb{P}\left\{Y_{T}=-T\right\}+f \mathbb{P}\left\{Y_{T}>=-T\right\}+\mathbb{P}\left\{Y_{T}=-T\right\}(-T-r-1) \\
& =f-(f+r+1) \mathbb{P}\left\{Y_{T}=-T\right\} \\
& <f .
\end{aligned}
$$

Thus, we have $C_{T+1}^{q} \leq f=C_{0}^{q}$ for all $T \geq 1$.

Next, for a more general $\bar{p}$, we have

Proposition 6. For general $\bar{p} \neq \frac{1}{2}$ and fixed values $r, f, p, q, C_{1}^{q}-C_{0}^{q}$ is an increasing linear function of $\bar{p}$. As a result, the optimal strategy is a threshold type, depending on the sign of $\left(C_{1}^{q}-C_{0}^{q}\right)(f, r, p, \bar{p})$, the optimal strategy is either using the market order or the best bid. There is at most one threshold of switching.

Proof. Let $C_{2, T-1}^{p}$ denote the expected cost of placing a limit bid order at the price of 2 tick lower than the current best ask price, with $T-1$ price changes left and $q=p$. Then $C_{2, T-1}^{p} \geq-2-r$, and

$$
C_{1}^{0}=(1-\bar{p})(-1-r)+\bar{p}\left(1+C_{2, T-1}^{p}\right),
$$

where $C_{2, n-1}^{p}$ is independent to $\bar{p}$ and $\left(1+C_{2, n-1}^{p}\right) \geq-1-r$. Thus $C_{1}^{0}$ is an increasing linear function of $\bar{p}$. Similarly for $C_{1}^{1}$. Then combining $C_{1}^{1}$ and $C_{1}^{0}$ we conclude that $C_{1}^{q}$ is linear of $\bar{p}$. Recall that $C_{0}^{q}=f$, then the result follows. Furthermore, $C_{1}^{0}=-1-r<0$ when $\bar{p}=0$, and $C_{1}^{0}=1+C_{2, T-1}^{p}$ when $\bar{p}=1$. Clearly this expression could be either positive or negative depending on the parameters.

\subsubsection{Analysis of the Optimal Placement Strategy: the Multi-period Case}

To be consistent and comparable with the analysis in the single-period case, we assume for the multiperiod analysis that after each price change at each time step $1, \cdots, T$, one can adjust the non-executed order with the following options:

- Cancel the non-executed order and replace it with a market order (denoted this action as Act $_{M}$ );

- Cancel the non-executed order and replace it with the best bid (denoted this action as $A c t_{L}$ );

- Cancel the non-executed order and do nothing, i.e. wait (denote this action as $A c t_{N}$ ).

Since the number of price changes remaining before the deadline is more critical to the analysis, in this section we use $s=T-t$ to denote the remaining time steps at time $t$. That is, $A_{s}$ is the best ask price when there are $s$ steps remaining. Moreover, we keep the same assumption that the limit bid order placed at price of $A_{s}-1$ will be executed with probability one if $A_{s-1}=A_{s}-1$, and will get executed with probability $q$ if $A_{s-1}=A_{s}+1$. 
Comparing the expected costs We will introduce some notations.

- $U_{s, A_{s}}^{L}$ is the expected cost per share with action $A c t_{L}$ with $s$ time steps remaining and conditioned on the previous price change being up;

- $U_{s, A_{s}}^{M}$ is the expected cost per share with action $A c t_{M}$ with $s$ time steps remaining and conditioned on the previous price change being up;

- $U_{s, A_{s}}^{N}$ is the expected cost per share action $A c t_{N}$ with $s$ time steps remaining and conditioned on the previous price change being up.

By symmetry, it is easy to see that

$$
U_{s, A_{s}}^{L}=A_{s}+U_{s, 0}^{L}
$$

Thus it suffices to focus on $U_{s, 0}^{L}$ and rewrite $U_{s}^{L}$ for $U_{s, 0}^{L}$ and similarly for $U_{s}^{M}$ and $U_{s}^{N}$, and define

$$
U_{s}:=\min \left\{U_{s}^{M}, U_{s}^{L}, U_{s}^{N}\right\}
$$

Similarly, we use $D_{s}^{M}, D_{s}^{L}, D_{s}^{N}$ as the expected costs for placing market order, placing limit order at the price of -1 , and placing no orders, conditioned on the previous price change being down, and define

$$
D_{s}:=\min \left\{D_{s}^{M}, D_{s}^{L}, D_{t}^{N}\right\}
$$

Clearly we have

Proposition 7. $U_{0}^{M}=U_{0}^{L}=U_{0}^{N}=D_{0}^{M}=D_{0}^{L}=D_{0}^{N}=f$. Moreover, for $1 \leq s \leq T$,

$$
\begin{aligned}
& U_{s}^{M}=D_{s}^{M}=f, \\
& U_{s}^{L}=(p q+1-p)(-1-r)+p(1-q)\left(1+U_{s-1}\right), \\
& U_{s}^{N}=(1-p)\left(-1+D_{s-1}\right)+p\left(1+U_{s-1}\right), \\
& D_{s}^{L}=(p+(1-p) q)(-1-r)+(1-p)(1-q)\left(1+U_{s-1}\right), \\
& D_{s}^{N}=p\left(-1+D_{s-1}\right)+(1-p)\left(1+U_{s-1}\right) .
\end{aligned}
$$

Furthermore,

Lemma 8. Both $D_{s}$ and $U_{s}$ are non-increasing functions with respect to $s$.

Proof. Let $S_{s}^{D}$ be the corresponding optimal trading strategy for the value function $D_{s}$. Then with $s+1$ time step remaining and $X_{s+1}=-1$, one can adopt the strategy $S_{s}^{D}$ for the first $s$ price changes until there is one more step remaining, and then use the market order directly. This will lead to an expected cost $D_{s}$. Since this is only one of all the possible strategies for this case, clearly $D_{s+1} \leq D_{s}$. Similarly $U_{s+1} \leq U_{s}$

Lemma 9. For $0 \leq s \leq T-1, f \geq U_{s} \geq-r-2+\frac{p}{(1-p)(p+q-p q)}, U_{s}^{L} \leq U_{s}^{M}, D_{s}^{L} \leq D_{s}^{N}$. That is, if the previous price change is going up, using the market order is never optimal; if the previous price change is going down, then one should never wait. 
Proof. By induction. For $s=0,-2-r+\frac{p}{(1-p)(p+q-p q)} \leq-2-r+\frac{p}{(1-p) p} \leq-r \leq f=U_{0}$. Notice that $D_{0}^{L}=D_{0}^{N}=f, U_{0}^{L}=U_{0}^{M}=f$, thus the claim holds for $s=0$.

Now suppose the lemma holds for all $k \leq s$, then for $s+1$ we have

$$
U_{s+1}^{M}=f \geq-2-r+\frac{p}{(1-p)(p+q-p q)}
$$

as we have already shown in the case $s=0$, and

$$
\begin{aligned}
U_{s+1}^{L} & =(p q+1-p)(-1-r)+p(1-q)\left(1+U_{s}\right) \\
& \geq-1-r+\frac{p^{2}(1-q)}{(1-p)(p+q-p q)} \\
& =-2-r+\frac{p+q-2 p q}{(1-p)(p+q-p q)} \\
& \geq-2-r+\frac{p}{(1-p)(p+q-p q) .}
\end{aligned}
$$

Meanwhile, $U_{s+1}^{N}=(1-p)\left(-1+\min \left\{D_{s}^{L}, D_{s}^{N}, D_{s}^{M}\right\}\right)+p\left(1+U_{s}\right)$. Note that

$$
\begin{aligned}
& (1-p)\left(-1+D_{s}^{L}\right)+p\left(1+U_{s}\right) \\
= & (1-p)\left(-1+(p+(1-p) q)(-1-r)+(1-p)(1-q)\left(1+U_{s-1}\right)\right)+p\left(1+U_{s}\right) \\
= & (1-p)\left(-1-1-r+(1-p)(1-q)\left(2+r+U_{s-1}\right)\right)+p\left(1+U_{s}\right) \\
\geq & -2-r+\frac{p(1-p)^{2}(1-q)+p^{2}}{(1-p)(p+q-p q)}+p \\
= & -2-r+\frac{p}{(1-p)(p+q-p q)},
\end{aligned}
$$

and

$$
\begin{aligned}
& (1-p)\left(-1+D_{s}^{M}\right)+p\left(1+U_{s}\right) \\
= & (1-p)(-1+f)+p\left(1+U_{s}\right) \\
\geq & 2 p-1+p U_{s} \geq 2 p-1-2 p-p r+\frac{p^{2}}{(1-p)(p+q-p q)} \\
\geq & -2-r+\frac{p}{(1-p)(p+q-p q)} .
\end{aligned}
$$

Since $D_{s}^{N} \geq D_{s}^{L},(1-p)\left(-1+D_{s}^{N}\right)+p\left(1+U_{s}\right) \geq(1-p)\left(-1+D_{s}^{L}\right)+p\left(1+U_{s}\right) \geq-2-r+\frac{p}{(1-p)(p+q-p q)}$. Hence $U_{s+1}^{N}=(1-p)\left(-1+D_{s}\right)+p\left(1+U_{s}\right) \geq-2-r+\frac{p}{(1-p)(p+q-p q)}$. Note that $U_{s}^{L}=(p q+1-p)(-1-$ $r)+p(1-q)\left(1+U_{s}\right) \leq p(1-q) U_{s} \leq f$. Therefore, when the previous price change is down,

$$
\begin{aligned}
D_{s+1}^{N} & =p\left(-1+D_{s}\right)+(1-p)\left(1+U_{s}\right) \\
& =D_{s+1}^{L}+p\left(r+D_{s}\right)+(1-p) q\left(2+r+U_{s}\right) \\
& =D_{s+1}^{L}+p\left(r+(p+(1-p) q)(-1-r)+(1-p)(1-q)\left(1+U_{s-1}\right)\right)+(1-p) q\left(2+r+U_{s}\right) \\
& \geq D_{s+1}^{L}+p\left(-1+(1-p)(1-q) \frac{p}{(1-p)(p+q-p q)}\right)+(1-p) q \frac{p}{(1-p)(p+q-p q)} \\
& =D_{s+1}^{L}-p+\frac{p^{2}(1-q)+p q}{p+q-p q}=D_{s+1}^{L} .
\end{aligned}
$$

Hence the lemma holds for $s+1$ and therefore for any $T-1 \geq s \geq 0$ by mathematical induction. 
Moreover, we see

Lemma 10. Knowing the previous price changes, the optimal strategy is the switching type:

- When the previous price change is up: there exists $s_{1}^{*} \in[1, \infty]$ such that for any $s \leq s_{1}^{*}$ using the best bid is optimal; and for any $s>s_{1}^{*}$ waiting is optimal.

- When the previous price change is going down: there exists $s_{2}^{*} \in[0, \infty]$, such that for any $s \leq s_{2}^{*}$ using market order is optimal; and for any $s>s_{2}^{*}$ using the best bid is optimal.

Proof. By Lemma 8 and Lemma 9, it suffices to consider

$$
\begin{aligned}
U_{s}^{L}-U_{s}^{N} & =(p q+1-p)(-1-r)+p(1-q)\left(1+U_{s-1}\right)-(1-p)\left(-1+D_{s-1}\right)+p\left(1+U_{s-1}\right) \\
& =-p q\left(2+r+U_{s-1}\right)-(1-p)\left(r+D_{s-1}\right) .
\end{aligned}
$$

This is a non-decreasing function with respect to $s$ for $s \geq 1$. Thus if $U_{s}^{L} \geq U_{s}^{N}$ for some $s_{1}^{*}$, then the inequality holds for any $s>s_{1}^{*}$. And $U_{1}^{L}-U_{1}^{N}=(1-p+p q)(-1-r-f)<0$ implying $s_{1}^{*} \geq 1$. The proof for the part with $s_{2}^{*}$ is similar.

We also find that

Lemma 11. $s_{1}^{*}>s_{2}^{*}$.

Proof. Note

$$
\begin{aligned}
U_{s}^{L}-U_{s}^{N} & =(p q+1-p)(-1-r)+p(1-q)\left(1+U_{s-1}\right)-(1-p)\left(-1+D_{s-1}\right)+p\left(1+U_{s-1}\right) \\
& =-p q\left(2+r+U_{s-1}\right)-(1-p)\left(r+D_{s-1}\right) .
\end{aligned}
$$

When $D_{s-1}^{M} \leq D_{s-1}^{L}$, we have $U_{s}^{L}-U_{s}^{N} \leq 0$, because $D_{s-1}=f \geq 0$.

Now, we are ready to state the optimal strategy.

Theorem 12. [Optimal placement strategy for $0<s \leq T-1$ ] Let $0<s \leq T-1$ represent the time remaining. There exist positive integers $s_{1}^{*}, s_{2}^{*}$ with $s_{1}^{*}>s_{2}^{*}>0$ such that

- for any $s>s_{1}^{*}$ : the optimal placement strategy is to wait if the previous price change is up, and to use the best bid if the previous price change is down;

- for any $s_{2}^{*}<s \leq s_{1}^{*}$ : it is optimal to use the best bid;

- for any $s \leq s_{2}^{*}$ : it is optimal to use the best bid order if the previous price change is going up, and to use the market order if the previous price change is down.

Moreover,

$$
s_{1}^{*}=\left\lceil[\log (p-p q)]^{-1} \log \frac{q(1-2 p)}{((1-p+p q)(f+2+r)-1)(1-q)\left(p^{2} q+1+p^{2}-2 p\right)}\right\rceil+1,
$$

and

$$
s_{2}^{*}=\left\lceil[\log (p-p q)]^{-1} \log \frac{(f+r+1)(1-p+p q)-(1-p)(1-q)}{(1-p)(1-q)((f+2+r)(1-p+p q)-1)}\right\rceil .
$$

(See Figure 1.) 


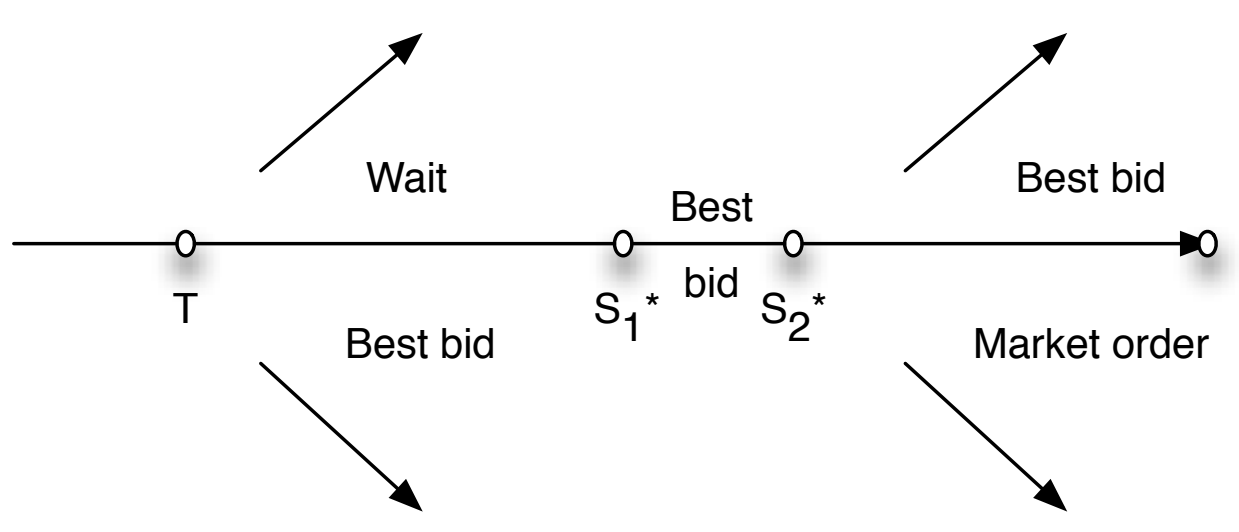

Figure 1: Illustration of the optimal trading strategy for $0<s \leq T-1$

Proof. The theorem is obvious from Lemma 9, Lemma 10, and lemma 11. In fact, one can calculate $s_{1}^{*}$ and $s_{2}^{*}$ explicitly as follows. For $s \leq s_{1}^{*}$ one can recursively calculate the sequence $U_{s}$ by the following equation and the boundary condition:

$$
\begin{aligned}
& U_{s}=p(1-q)\left(U_{s-1}+1\right)+(1-p+p q)(-1-r) \text { for } s_{1}^{*} \geq s \geq 1, \\
& U_{0}=f .
\end{aligned}
$$

Solving it we get

$$
\begin{aligned}
U_{s} & =(p-p q)^{s}\left(f+\frac{2 p-2 p q-1-r(1-p+p q)}{p-1-p q}\right)+\frac{2 p-2 p q-1-r(1-p+p q)}{1-p+p q} \\
& =(p-p q)^{s}\left(f+2+r-\frac{1}{1-p+p q}\right)-2-r+\frac{1}{1-p+p q} \quad \text { for } \quad s_{1}^{*} \geq s \geq 0 .
\end{aligned}
$$

Now that $s=s_{1}^{*}, D_{s_{1}^{*}}^{L} \leq D_{s_{1}^{*}}^{M}$, implying $D_{s_{1}^{*}}=D_{s_{1}^{*}}^{L}$ and

$$
\begin{aligned}
U_{s_{1}^{*}+1}^{L}-U_{s_{1}^{*}+1}^{N}= & -p q\left(2+r+U_{s_{1}^{*}}\right)-(1-p)\left(r+D_{s_{1}^{*}}\right) \\
= & -p q\left(2+r+U_{s^{*}}\right)-(1-p)\left(r+D_{s_{1}^{*}}^{L}\right) \\
= & -p q\left(2+r+(p-p q)^{s_{1}^{*}}\left(f+2+r-\frac{1}{1-p+p q}\right)-2-r+\frac{1}{1-p+p q}\right) \\
& -(1-p)\left(r+(p+(1-p) q)(-1-r)+(1-p)(1-q)\left(1+U_{s_{1}^{*}-1}\right)\right) \\
= & -p q\left((p-p q)^{s_{1}^{*}}\left(f+2+r-\frac{1}{1-p+p q}\right)+\frac{1}{1-p+p q}\right) \\
& -(1-p)(r+(p+(1-p) q)(-1-r) \\
& \left.+(1-p)(1-q)\left(1+(p-p q)^{s_{1}^{*}-1}\left(f+2+r-\frac{1}{1-p+p q}\right)-2-r+\frac{1}{1-p+p q}\right)\right) \\
= & \frac{q(1-2 p)}{1-p+p q}-\left(f+2+r-\frac{1}{1-p+p q}\right)\left((p-p q)^{s_{1}^{*}} p q+(p-p q)^{s_{1}^{*}-1}(1-p)^{2}(1-q)\right) \\
= & \frac{q(1-2 p)}{1-p+p q}-\left(f+2+r-\frac{1}{1-p+p q}\right)(p-p q)^{s_{1}^{*}-1}(1-q)\left(p^{2} q+1+p^{2}-2 p\right) \geq 0 .
\end{aligned}
$$


Thus

$$
s_{1}^{*}=\left\lceil[\log (p-p q)]^{-1} \log \frac{q(1-2 p)}{((1-p+p q)(f+2+r)-1)(1-q)\left(p^{2} q+1+p^{2}-2 p\right)}\right\rceil+1 .
$$

Simple calculation confirms that this above expression for $s_{1}^{*}$ is indeed no less than 1. Moreover, for $s \geq s_{1}^{*}$ we have

$$
\begin{aligned}
U_{s+1} & =(1-p)\left(-1+D_{t}\right)+p\left(1+U_{s}\right) \\
& =p U_{s}+2 p-1+(1-p)\left[(1-p-q+p q)\left(1+U_{s-1}\right)+(-1-r)(p+q-p q)\right] \\
& =p U_{s}+(1-p)(1-p-q+p q) U_{t-1}+2 p-1-(1+r)(1-p)(p+q-p q) \\
& =a_{1} U_{s}+a_{2} U_{s-1}+a_{3}
\end{aligned}
$$

where $a_{1}=p, a_{2}=(1-p)(1-p-q+p q), a_{3}=2 p-1-(1+r)(1-p)(p+q-p q)$. And the initial condition for the iteration is

$$
\begin{gathered}
U_{s *}=(p-p q)^{s_{1}^{*}}\left(f+2+r-\frac{1}{1-p+p q}\right)-2-r+\frac{1}{1-p+p q}, \\
U_{s^{*}-1}=(p-p q)^{s_{1}^{*}-1}\left(f+2+r-\frac{1}{1-p+p q}\right)-2-r+\frac{1}{1-p+p q} .
\end{gathered}
$$

In order to derive an explicit formula for $U_{t}$, we need to rewrite it as

$$
U_{s+1}-a_{4}=a_{1}\left(U_{s}-a_{4}\right)+a_{2}\left(U_{s-1}-a_{4}\right),
$$

where $a_{4}=\frac{a_{3}}{1-a_{1}-a_{2}}$. Solving this, we see

$$
U_{s}-a_{4}=A\left(\frac{a_{1}+\sqrt{a_{1}^{2}+4 a_{2}}}{2}\right)^{s-s_{1}^{*}-1}+B\left(\frac{a_{1}+\sqrt{a_{1}^{2}-4 a_{2}}}{2}\right)^{s-s_{1}^{*}-1},
$$

where $A$ and $B$ satisfy the following linear equation systems:

$$
\begin{aligned}
A+B & =a_{1} U_{s_{1}^{*}}+a_{2} U_{s_{1}^{*}}+a_{3}-a_{4}, \\
A \frac{a_{1}+\sqrt{a_{1}^{2}+4 a_{2}}}{2}+B \frac{a_{1}-\sqrt{a_{1}^{2}+4 a_{2}}}{2} & =\left(a_{1}^{2}+a_{2}\right) U_{s_{1}^{*}}+a_{1} a_{2} U_{s_{1}^{*}-1}+a_{1} a_{3}+a_{3}-a_{4} .
\end{aligned}
$$

Now we can compute $s_{2}^{*}$, which is the largest integer satisfying the following inequality

$$
(1-p)(1-q)\left[(p-p q)^{s}\left(f+r+2-\frac{1}{1-p+p q}\right)+\frac{1}{1-p+p q}\right]-r-1 \geq f,
$$

and get

$$
s_{2}^{*}=\left\lceil[\log (p-p q)]^{-1} \log \frac{(f+r+1)(1-p+p q)-(1-p)(1-q)}{(1-p)(1-q)((f+2+r)(1-p+p q)-1)}\right\rceil
$$

One can verify from the expression of $s_{2}^{*}$ that it is non-negative.

Finally one can calculate $D_{s}$ as follows

$$
D_{s}=\left\{\begin{array}{l}
f, \quad s \leq s_{2}^{*} ; \\
(p+(1-p) q)(-1-r)+(1-p)(1-q)\left(1+U_{s-1}\right), \quad s>s_{2}^{*} .
\end{array}\right.
$$

Our last step is to derive the optimal strategy for the special case $s=T$. 
Theorem 13 (Threshold-type optimal strategy for $s=T$ ). There exist up to two thresholds $\bar{p}_{1}^{*}$ and $\bar{p}_{2}^{*}$, with $\bar{p}_{1}^{*} \leq \bar{p}_{2}^{*} \in[0,1]$, which separate $[0,1]$ into up to 3 intervals. Each interval corresponds to one of the actions: waiting/doing nothing, using the best bid orders, and using the market orders. In particular, the potential thresholds are from the following set:

$$
\left\{\frac{D_{T-1}+r}{D_{T-1}+(1-q) r-2 q-q U_{T-1}}, \quad \frac{f+r+1}{(1-q)\left(2+r+U_{T-1}\right)}, \quad \frac{f+1-D_{T-1}}{U_{T-1}+2-D_{T-1}}\right\} .
$$

Furthermore, when $\bar{p}=0$, placing best bid orders is always better than market orders, and when $\bar{p}=1$, placing best bid orders is always better than to wait.

Proof. Let $\operatorname{Cost}^{L}(T, \bar{p})$ be the expected cost with placing the best bid order with $T$ steps left and the initial probability of the first price change being up is $\bar{p}$. Similarly we define $\operatorname{Cost}^{N}(T, \bar{p})$ and $\operatorname{Cost}^{M}(T, \bar{p})$ for placing no orders and using market orders, respectively. Then

$$
\begin{aligned}
\operatorname{Cost}^{L}(T, \bar{p}) & =(1-\bar{p}+\bar{p} q)(-1-r)+(\bar{p}-\bar{p} q)\left(1+U_{T-1}\right) \\
& =\bar{p}(1-q)\left(2+r+U_{T-1}\right)-1-r \\
\operatorname{Cost}^{N}(T, \bar{p}) & =(1-\bar{p})\left(-1+D_{T-1}\right)+\bar{p}\left(1+U_{T-1}\right) \\
& =\bar{p}\left(U_{T-1}+2-D_{T-1}\right)-1+D_{T-1}, \\
\operatorname{Cost}^{M}(T, \bar{p}) & =f .
\end{aligned}
$$

Here $\operatorname{Cost}^{M}(T, \bar{p})$ is a constant, $\operatorname{Cost}^{L}(T, \bar{p})$, and $\operatorname{Cost}^{N}(T, \bar{p})$ are linear functions of $\bar{p}$. It is easy to check their first order coefficients are positive as

$$
\begin{aligned}
&(1-q)\left(2+r+U_{T-1}\right) \geq(1-q)\left(2+r-2-r+\frac{p}{(1-p)(p+q-p q)}\right. \\
&=(1-q) \frac{p}{(1-p)(p+q-p q)}>0, \\
& U_{T-1}+2-D_{T-1} \geq 2+\min \left\{U_{T-1}^{L}-D_{T-1}^{L}, U_{T-1}^{N}-D_{T-1}^{N}, U_{T-1}^{M}-D_{T-1}^{M}\right\} \\
&=2+\min \left\{(1-2 p-q+2 p q)\left(-2-r-U_{T-2}\right),(1-2 p)\left(-2+D_{T-2}-U_{T-2}\right), 0\right\} .
\end{aligned}
$$

And since $(1-2 p-q+2 p q)<1$ and $-2-r-U_{T-2} \geq-\frac{p}{(1-p)(p+q-p q)}>-\frac{1}{1-p}>-2$, thus $-2+(1-$ $2 p-q+2 p q)\left(-2-r-U_{T-2}\right)>0$. Note also $D_{T-2} \geq U_{T-2}$. Thus $(1-2 p)\left(-2+D_{T-2}-U_{T-2}\right)>-2$. Therefore $U_{T-1}+2-D_{T-1}>0$. Thus $\operatorname{Cost}^{L}(T, \bar{p})$ and $\operatorname{Cost}^{N}(T, \bar{p})$ are increasing functions. It follows then there are up to 3 intersections and each intersection represents the switch between two actions. Since one of the intersection is the switch between the action with the highest expected cost and the action with the second highest cost, these intersections could be beyond the range of $[0,1]$. Hence there may be up to 2 thresholds. On each interval, one of the action is optimal. Furthermore, the (possible) thresholds are from the set of the intersections:

$$
\left\{\frac{D_{T-1}+r}{D_{T-1}+(1-q) r-2 q-q U_{T-1}}, \quad \frac{f+r+1}{(1-q)\left(2+r+U_{T-1}\right)}, \quad \frac{f+1-D_{T-1}}{U_{T-1}+2-D_{T-1}}\right\} .
$$

And $\operatorname{Cost}^{L}(T, 0)=-1-r<f=\operatorname{Cost}^{M}(T, 0)$, thus placing best bid orders are better than placing market orders when $\bar{p}=0$. Finally, when $\bar{p}=1$, placing best bid orders is better than waiting because

$$
\begin{aligned}
\operatorname{Cost}^{L}(T, 1) & =(1-q)\left(2+r+U_{T-1}\right)-1-r \\
& \leq 2+r+U_{T-1}-1-r \\
& =\left(U_{T-1}+2-D_{T-1}\right)-1+D_{T-1} \\
& =\operatorname{Cost}^{N}(T, 1) .
\end{aligned}
$$



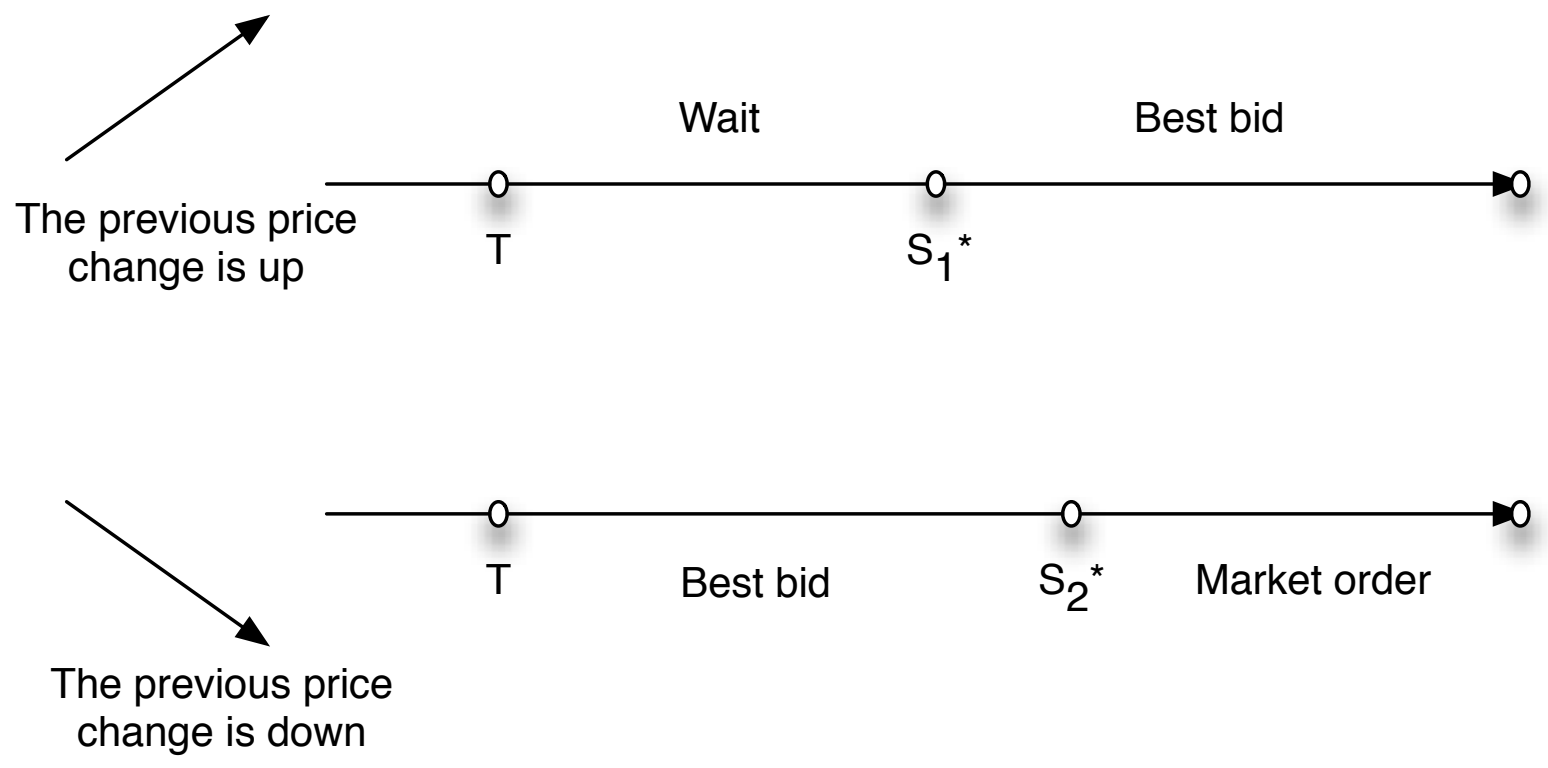

Figure 2: Illustration of the optimal trading strategy

Remark 2. The threshold-type optimal strategy is expected given the Markov property of $\left(A_{t}, A_{t-1}\right)$ or equivalently that of $\left(A_{t}, X_{t}\right)$. The optimal strategy is in fact independent of $A_{t}$. This is not very surprising because in the optimal placement problem, the best strategy is derived from comparing choices of the best bid, the market order or no action at any given price level $A_{s}$, hence the absolute expected value of each strategy is less relevant. (See Figure 2.)

Remark 3. For the degenerate case of $p=\bar{p}=\frac{1}{2}$, then checking with of the above analysis reveals that it is always optimal to peg the bid.

Remark 4. If we let $T=1$, then both the single-period model and multi-period model yield the same trading strategy. To see this, we see that for the single period model, the best bid order is used if

$$
\bar{p}<\frac{r+f+1}{(r+f+2)(1-q)}
$$

It implies that when $r+f=0$ and $q=0$, we choose the best bid order if the probability of price going up is less than $1 / 2$. And for the multi-period model, we have

$$
\begin{aligned}
\operatorname{Cost}^{L}(1, \bar{p}) & =\bar{p}(1-q)(2+r+f)-1-r, \\
& \leq 2 \bar{p}-1+f, \\
& =\operatorname{Cost}^{N}(1, \bar{p}) .
\end{aligned}
$$

Thus we only choose to place market orders or the best bid orders. And it is easy to see that when $\bar{p}<\frac{r+f+1}{(r+f+2)(1-q)}$, the best bid order is better than the market order, and vice versa. This is consistent with the single period model. 


\subsection{A Model with Price Impact}

In the previous model, we assume no price impact from any market or limit orders. In this section, we will add price impact factor on both limit orders and market orders.

To make the analysis more feasible, our model will focus on the limit order book with market orders and the best limit orders, and assume that the price impact of market and limit orders is linear and additive. These assumptions are consistent with the empirical study of Cont, Kukanov, and Stoikov (2013) [19] as well as the modelling framework of optimal execution problems with price impact.

Dynamics of the price model and analysis. Let $A_{t}$ be the best ask price at time $t,\left\{\epsilon_{t}\right\}_{t=1,2, \cdots, T}$ is an i.i.d. random sequence with mean 0 . Then the dynamics of $A_{t}$ is modeled as:

$$
A_{t+1}=A_{t}+c_{1} m_{t}+c_{2} I_{t} l_{t}+\epsilon_{t+1}
$$

with $A_{0}=0$,

$$
I_{t}= \begin{cases}1 & \text { if the limit order was executed by time } t \\ 0 & \text { if the limit order was not executed by time } t\end{cases}
$$

Here $c_{1}$ and $c_{2}$ are the price impact factor for market orders and limit orders, and $m_{t}$ and $l_{t}$ are the order sizes placed at time $t$ for market orders and limit orders, respectively.

Now, take $r$ the rebate for an executed limit order and $f$ the transaction fee for a market order as before. At each time, a limit order will be executed with probability one at the price of 1 tick lower than $A_{t}$. Note that independent of whether the limit order is executed or not, the transaction price for a market order and limit order at time $t$ will be $A_{t}+c_{1} m_{t}+c_{2} l_{t}+\epsilon_{t}+f$ and $A_{t}+c_{1} m_{t}+c_{2} l_{t}+\epsilon_{t}-1-r$, correspondingly. For simplification and ease of exposition, and without much loss of generality, we assume that $c_{1}=c_{2}=c$. In reality, it has been observed that $c_{1}>c_{2}$ since the market order will affect the trading directly and immediately while a large limit order may take longer time. But this differentiation of $c_{1}$ and $c_{2}$ will not change much of the structural results of our analysis and we opt for clarity of exposition.

Clearly, because of the price impact, the reduction of a general $N$ to $N=1$ is no longer valid. Now Eqn. (2.13) is rewritten as

$$
A_{t+1}=A_{t}+c\left(m_{t}+I_{t} l_{t}\right)+\epsilon_{t+1} .
$$

Moreover, we assume that the probability of the limit orders being executed is $p_{l}$.

Let $C_{t}\left(n_{t}, m_{t}, l_{t}, A_{t}\right)$ be the expected cost at time $t$ with current price of $A_{t}, 0 \leq n_{t} \leq N$ shares left to purchase, placing limit order of $l_{t}$ and market order of $m_{t}$. Let $V_{t}\left(n_{t}, A_{t}\right)$ be the expected cost after optimization over $m_{t}$ and $l_{t}$. Then according to the dynamic programming principle, the optimal placement problem can be formulated as

$$
\begin{gathered}
V_{t}\left(n_{t}, A_{t}\right)=\min _{\substack{\leq m_{t}, l_{t} \\
m_{t}+l_{t} \leq n_{t}}} C_{t}\left(n_{t}, m_{t}, l_{t}, A_{t}\right), \quad 1 \leq t \leq T-1, \\
V_{T}\left(n_{T}, A_{T}\right)=n_{T}\left(f+A_{T}+c n_{T}\right) .
\end{gathered}
$$

subject to Eqn. (2.14), with

$$
\begin{aligned}
C_{t}\left(n_{t}, m_{t}, l_{t}, A_{t}\right)= & \mathbb{E}\left\{m_{t}\left(f+A_{t}+c\left(m_{t}+l_{t}\right)+\epsilon_{t}\right)+p_{l} l_{t}\left(A_{t}-r-1+c\left(m_{t}+l_{t}\right)+\epsilon_{t}\right)\right. \\
& \left.+p_{l} V_{t+1}\left(n_{t}-m_{t}-l_{t}, A_{t}+c\left(m_{t}+l_{t}\right)+\epsilon_{t}\right)+\left(1-p_{l}\right) V_{t+1}\left(n_{t}-m_{t}, A_{t}+c m_{t}+\epsilon_{t}\right)\right\} .
\end{aligned}
$$

By mathematical induction, it is easy to check the following lemma 
Lemma 14. For $0 \leq t \leq T-1$,

$$
C_{t}\left(n_{t}, m_{t}, l_{t}, x\right)=C_{t}\left(n_{t}, m_{t}, l_{t}, 0\right)+x n_{t},
$$

and

$$
V_{t}\left(n_{t}, x\right)=V_{t}\left(n_{t}, 0\right)+x n_{t} .
$$

Proof. First, this lemma is valid for $t=T-1$ since

$$
\begin{aligned}
& C_{T-1}\left(n_{T-1}, m_{T-1}, l_{T-1}, A_{T-1}\right) \\
= & \mathbb{E}\left\{m_{T-1}\left(f+A_{T-1}+c\left(m_{T-1}+l_{T-1}\right)+\epsilon_{T-1}\right)+p_{l} l_{T-1}\left(A_{T-1}-r-1+c\left(m_{T-1}+l_{T-1}\right)+\epsilon_{T-1}\right)\right. \\
& +p_{l}\left(n_{T-1}-m_{T-1}-l_{T-1}\right)\left(A_{T-1}+c\left(m_{T-1}+l_{T-1}\right)+c\left(n_{T-1}-m_{T-1}-l_{T-1}\right)+\epsilon_{T-1}+\epsilon_{T}\right) \\
& \left.+\left(1-p_{l}\right)\left(n_{t}-m_{t}\right)\left(A_{t}+c m_{t}+c\left(n_{T-1}-m_{T-1}\right)+\epsilon_{T-1}+\epsilon_{T}\right)\right\} \\
= & m_{T-1}\left(f+A_{T-1}+c\left(m_{T-1}+l_{T-1}\right)\right)+p_{l} l_{T-1}\left(A_{T-1}-r-1+c\left(m_{T-1}+l_{T-1}\right)\right) \\
& +p_{l}\left(n_{T-1}-m_{T-1}-l_{T-1}\right)\left(A_{T-1}+c n_{T-1}\right)+\left(1-p_{l}\right)\left(n_{T-1}-m_{T-1}\right)\left(A_{T-1}+c n_{T-1}\right) \\
= & n_{T-1} A_{T-1}+m_{T-1}\left(f+c\left(m_{T-1}+l_{T-1}\right)\right)+p_{l} l_{T-1}\left(-r-1+c\left(m_{T-1}+l_{T-1}\right)\right) \\
& +p_{l}\left(n_{T-1}-m_{T-1}-l_{T-1}\right)\left(c n_{T-1}\right)+\left(1-p_{l}\right)\left(n_{T-1}-m_{T-1}\right)\left(c n_{T-1}\right) \\
= & n_{T-1} A_{T-1}+C_{T-1}\left(n_{T-1}, m_{T-1}, l_{T-1}, 0\right) .
\end{aligned}
$$

And because the coefficient of $A_{T-1}$ is $n_{T-1}$,

$$
\begin{aligned}
V_{T-1}\left(n_{T-1}, A_{T-1}\right) & =\min C_{T-1}\left(n_{T-1}, m_{T-1}, l_{T-1}, A_{T-1}\right) \\
& =n_{T-1} A_{T-1}+\min C_{T-1}\left(n_{T-1}, m_{T-1}, l_{T-1}, 0\right) \\
& =n_{T-1} A_{T-1}+V_{T-1}\left(n_{T-1}, 0\right) .
\end{aligned}
$$

Thus the assertion holds for $k=T-1$. Assume that this is true for some $k=t+1$ with $t \geq 0$, then for $k=t$,

$$
\begin{aligned}
C_{t}\left(n_{t}, m_{t}, l_{t}, A_{t}\right) & \\
= & \mathbb{E}\left\{m_{t}\left(f+A_{t}+c\left(m_{t}+l_{t}\right)+\epsilon_{t}\right)+p_{l} l_{t}\left(A_{t}-r-1+c\left(m_{t}+l_{t}\right)+\epsilon_{t}\right)\right. \\
& \left.+p_{l} V_{t+1}\left(n_{t}-m_{t}-l_{t}, A_{t}+c\left(m_{t}+l_{t}\right)+\epsilon_{t}\right)+\left(1-p_{l}\right) V_{t+1}\left(n_{t}-m_{t}, A_{t}+c m_{t}+\epsilon_{t}\right)\right\} \\
= & m_{t}\left(f+A_{t}+c\left(m_{t}+l_{t}\right)\right)+p_{l} l_{t}\left(A_{t}-r-1+c\left(m_{t}+l_{t}\right)\right) \\
& +\mathbb{E}\left\{p_{l}\left[V_{t+1}\left(n_{t}-m_{t}-l_{t}, 0\right)+\left(n_{t}-m_{t}-l_{t}\right)\left(A_{t}+c\left(m_{t}+l_{t}\right)+\epsilon_{t}\right)\right]\right\} \\
& +\mathbb{E}\left\{\left(1-p_{l}\right)\left[V_{t+1}\left(n_{t}-m_{t}, 0\right)+\left(n_{t}-m_{t}\right)\left(A_{t}+c m_{t}+\epsilon_{t}\right)\right\}\right. \\
= & n_{t} A_{t}+C_{t}\left(n_{t}, m_{t}, l_{t}, 0\right) .
\end{aligned}
$$

And

$$
\begin{aligned}
V_{t}\left(n_{t}, A_{t}\right) & =\min C_{t}\left(n_{t}, m_{t}, l_{t}, A_{t}\right) \\
& =n_{t} A_{t}+\min C_{t}\left(n_{t}, m_{t}, l_{t}, 0\right) . \\
& =n_{t} A_{t}+V_{t}\left(n_{t}, 0\right) .
\end{aligned}
$$

Thus we see that the lemma holds for $k=t$ if it holds for $k=t+1$. By the recursive induction, the lemma is valid for $0 \leq t \leq T-1$.

This implies that the influence of the current price is a linear shift to the total cost and will not affect the optimization over $m_{t}$ and $l_{t}$. Thus

$$
\left(M_{t}, L_{t}\right)=\arg \min _{\substack{0 \leq m_{t}, l_{t} \\ m_{t}+l_{t} \leq n_{t}}} C_{t}\left(m_{t}, l_{t}, n_{t}, A_{t}\right)
$$

is independent of $A_{t}$. 
Properties of the optimal strategy for period $t$. The above analysis suggests that $M_{t}$ and $L_{t}$ are functions of $T-t$ and $n_{t}$. To make notations simpler, let $a=\frac{r+f+1}{c}$ be the normalized difference between market order and limit order and write $C_{t}\left(n_{t}, m_{t}, l_{t}\right)$ and $V_{t}\left(n_{t}\right)$ for $C_{t}\left(n_{t}, m_{t}, l_{t}, r+1\right)$ and $V_{t}\left(n_{t}, r+1\right)$, respectively. We see for $0 \leq t \leq T-1$,

$$
\begin{aligned}
C_{t}\left(n_{t}, m_{t}, l_{t}\right)= & m_{t}\left(m_{t}+l_{t}+a\right)+p_{l} l_{t}\left(m_{t}+l_{t}\right)+p_{l}\left[V_{t+1}\left(n_{t}-m_{t}-l_{t}\right)+\left(m_{t}+l_{t}\right)\left(n_{t}-m_{t}-l_{t}\right)\right] \\
& +\left(1-p_{l}\right)\left[V_{t+1}\left(n_{t}-m_{t}\right)+m_{t}\left(n_{t}-m_{t}\right)\right] \\
= & a m_{t}+n_{t} m_{t}+p_{l} n_{t} l_{t}+(1-p) m_{t} l_{t}+p_{l} V_{t+1}\left(n_{t}-m_{t}-l_{t}\right)+(1-p) V_{t+1}\left(n_{t}-m_{t}\right)
\end{aligned}
$$

Theorem 15. For any $t$ and $n_{t}$, we have $M_{t} \cdot L_{t}=0$. That is, the optimal strategy involves either market order or limit order at each step but never both. Moreover, $V_{t}\left(n_{t}\right)$ is a piece-wise quadratic function of $n_{t}$ with the second order coefficients within $(1 / 2,1]$.

Proof. The proof is by mathematical induction.

First, for $t=T, M_{T}=n_{T}$ and $L_{T}=0$. Thus $V_{T}\left(n_{T}\right)=n_{T}^{2}+a n_{T}$.

Second, suppose the theorem holds for $t+1$, and $z_{1}$ is the second order coefficient of $V_{t+1}\left(n_{t+1}\right)$ within $(1 / 2,1]$. Note that $V_{t+1}$ is a piece-wise quadratic function, $z_{1}$ depends on $n_{t+1}$ and piecewise constant. Thus the Hessian of $C_{t}$ is

$$
\mathbb{H}\left(C_{t}\right)=\left(\begin{array}{cc}
2 z_{1} & 1-p_{l}+2 z_{1} p \\
1-p_{l}+2 z_{1} p_{l} & 2 z_{1} p_{l}
\end{array}\right)
$$

Since $z_{1}>0$ and $4 z_{1}^{2}-\left(1-p_{l}+2 z_{1} p_{l}\right)=-\left(1-p_{l}\right)^{2}-\left(1-p_{l}\right)\left(1-z_{1}\right)<0$, meaning the Hessian of $C_{t}$ is neither semi-positive nor semi-negative. Thus the possible minimum value will only reach on the boundary. Notice that $V_{t+1}$ is a piece-wise quadratic function, we also need to consider the pairs of $\left(m_{t}, l_{t}\right)$ which makes $\left(n_{t}-m_{t}-l_{t}\right)$ or $\left(n_{t}-m_{t}\right)$ locates on the thresholds of $V_{t+1}$. Suppose $A$ is a threshold of $V_{t+1}$ and $m_{t}+l_{t}=A$. Then

$$
C_{t}^{\prime \prime}\left(l_{t}\right)=-2\left(1-p_{l}\right)+2\left(1-p_{l}\right) z_{1} \leq 0
$$

implying that $L_{t}=0, M_{t}=A$ or $L_{t}=A, M_{t}=0$. Similarly, we can show that in the case of $L_{t}=A$ or $M_{t}=A, C_{t}$ will be a quadratic function with a negative second order coefficient. Thus $M_{t} \cdot L_{t}=0$ for $t$.

Next, let us calculate $V_{t}$. In the case of $l_{t}=0$,

$$
C_{t}=a m_{t}+n_{t} m_{t}+V_{t+1}\left(n_{t}-m_{t}\right)
$$

which is a piece-wise quadratic function of $m_{t}$. On each piece, if not on the boundary of that piece, the optimal solution $M_{t}$ is a linear function of $n_{t}$ with coefficient $a_{1}=1-1 /\left(2 z_{1}\right)$, which is in the range of $(0,1 / 2]$. Then the second order coefficient of $V_{t}$ is $z_{1}\left(1-a_{1}\right)^{2}+a_{1}^{2}<1$. And if $M_{t}$ is on the boundary of that piece, then $M_{t}$ is still a linear function of $n_{t}$ with the first order coefficient of 1 . Then the second order coefficient of $V_{t}$ is $z_{1}\left(1-a_{1}\right)^{2}+a_{1}^{2}=1$. Similarly for the case of $m_{t}=0$,

$$
C_{t}=p_{l} n_{t} l_{t}+p_{l} V_{t+1}\left(n_{t}-l_{t}\right)+\left(1-p_{l}\right) V_{t+1}\left(n_{t}\right) .
$$

Therefore $L_{t}$ is a linear function of $n_{t}$ with the first order coefficient no greater than 1 , denoted by $b_{1}$. Then the second order coefficient of $V_{t}$ is $p_{l} z_{1}\left(1-b_{1}\right)^{2}+p_{l} b_{1}^{2}+\left(1-p_{l}\right) z_{1} \leq 1$. Therefore the theorem holds for $t$. By the mathematical induction, it holds for all $t \leq T$. 


\subsection{Optimal Placement vs. Optimal Execution: Connection to Bertsimas and Lo (1998) [10]}

The study of the optimal execution problem is pioneered in Bertsimas and Lo (1998) [10, where the stock price is modelled by a simple random walk with an additive impact of large sales. An intriguing consequence of this modelling approach is that the optimal strategy turns out to be more or less static or deterministic. That is, evenly dividing the total number of shares $N$ over the $T$ selling period is optimal.

Obviously, specifying $p_{l}=1$ or $p_{l}=0$ will reduce our model with price impact to the model of Bertsimas and Lo. In fact, we can show that in this special case the optimal strategy is the same as theirs. Indeed in the case of $p_{l}=1$ meaning the limit order will be executed for sure, then the market order is always dominated by the limit order. In Equation (2.17), substitute $p_{l}$ with 1, and use limit orders at the last period, we get

$$
\begin{aligned}
V_{T-1}\left(x, A_{T-1}\right)= & \min _{\substack{0 \leq m_{T-1}, l_{T-1} \\
m_{T-1}+l_{T-1} \leq x}}\left\{l_{T-1}\left(-1-r+c\left(m_{T-1}+l_{T-1}\right)+A_{T-1}\right)+\left(x-m_{T-1}-l_{T-1}\right)\left(x c+A_{T-1}-r-1\right)\right. \\
& \left.+m_{T-1}\left(f+c\left(m_{T-1}+l_{T-1}\right)+A_{T-1}\right)\right\} \\
= & \min _{\substack{0 \leq m_{T-1}, l_{T-1} \\
m_{T-1}+l_{T-1} \leq x}}\left\{c\left(m_{T-1}+l_{T-1}\right)^{2}-(c x-r-f-1)\left(m_{T-1}+l_{T-1}\right)-(r+f+1) l_{T-1}\right. \\
& \left.+x\left(A_{T-1}+c x-r-1\right)\right\} .
\end{aligned}
$$

Since $\left(m_{T-1}, l_{T-1}\right)$ is always dominated by $\left(0, m_{T-1}+l_{T-1}\right)$ if both of them are feasible, we have $M_{T-1}=0$, and

$$
V_{T-1}\left(x, A_{T-1}\right)=\min _{0 \leq l_{T-1} \leq x} c l_{T-1}^{2}-c x l_{T-1}+x\left(A_{T-1}+c x-r-1\right),
$$

whose minimum is of $3 c x^{2} / 4+x\left(A_{T-1}-r-1\right)$ at $l_{T-1}=\frac{x}{2}$. Inductively, suppose $\left(M_{t+1}=0, L_{t+1}=x /(T-t)\right)$ is the optimal solution for the period $t, 0 \leq t \leq T-1$ and $V_{t+1}\left(x, A_{t+1}\right)=c x^{2}(1+1 /(T-t)) / 2+x\left(A_{t+1}-\right.$ $r-1)$. Then at time $t$,

$$
\begin{aligned}
C_{t}\left(x, m_{t}, l_{t}, A_{t}\right)= & m_{t}\left(A_{t}+c\left(m_{t}+l_{t}\right)+f\right)+l_{t}\left(A_{t}+c\left(m_{t}+l_{t}\right)-r-1\right) \\
& +c\left(x-m_{t}-l_{t}\right)^{2}(1+1 /(T-t)) / 2+\left(x-m_{t}-l_{t}\right)\left(A_{t}+c\left(m_{t}+l_{t}\right)-r-1\right) \\
= & \frac{T-t+1}{2(T-t)} c\left(m_{t}+l_{t}\right)^{2}-\frac{1}{T-t}(c x-r-f-1)\left(m_{t}+l_{t}\right)+\frac{T-t+1}{2(T-t)} c x^{2} \\
& +x\left(A_{t}-r-1\right)-(r+f+1) l_{t} .
\end{aligned}
$$

Thus, for any feasible solution $\left(m_{t}, l_{t}\right),\left(0, m_{t}+l_{t}\right)$ is always better if $m_{t}>0$. Hence $M_{t}=0$. Further simple calculation concludes that

$$
\begin{aligned}
L_{t} & =\frac{x}{T-t+1}, \\
E_{t}\left(x, A_{t}\right) & =c x^{2}(1+1 /(T-t+1)) / 2+x\left(A_{t}-r-1\right) .
\end{aligned}
$$

Thus by mathematical induction, the optimal strategy is to always use limit orders, and if there are $T$ periods in total, then each time one places $1 / T$ of the total volume. If $p_{l}=0$, which means the limit order will not be executed, then similarly one can show that the optimal trading strategy is to use market orders only and to equally divide the total volume for each period. 
Example 1. To get a sense of the computational complexity, we give some illustration for $t=T-1$ with an explicit optimal strategy. Clearly,

$$
\begin{aligned}
V\left(x, A_{T-1}\right)= & \min _{\substack{0 \leq m_{T-1}, l_{T-1} \\
m_{T-1}+l_{T-1} \leq x}} \\
& m_{T-1}\left(f+c\left(m_{T-1}+l_{T-1}\right)+A_{T-1}\right) \\
& +p_{l}\left[l_{T-1}\left(-1-r+c\left(m_{T-1}+l_{T-1}\right)+A_{T-1}\right)+\left(x-m_{T-1}-l_{T-1}\right)\left(f+x c+A_{T-1}\right)\right] \\
& +\left(1-p_{l}\right)\left(x-m_{T-1}\right)\left(f+x c+A_{T-1}\right) .
\end{aligned}
$$

For any fixed $x$ and $A_{T-1}$, the RHS of (2.17) is a quadratic function of $m_{t-1}$ and $l_{t-1}$, whose Hessian is

$$
\left(\begin{array}{cc}
2 & p_{l}+1 \\
p_{l}+1 & 2 p_{l}
\end{array}\right)
$$

whose determinant is $-\left(p_{l}-1\right)^{2}<0$ for $p_{l}<1$. Clearly this Hessian is neither positive definite nor negative definite for all $m_{t-1}$ and $l_{t-1}$. Thus the function will reach its minimum only on the boundary. Moreover,

- If $p_{l}<\frac{1}{4}$, then there are two scenarios:

1. For $0 \leq x \leq 4 p_{l} \frac{r+f+1}{c}$, then $M_{T-1}=0, L_{T-1}=x$;

2. For $4 p_{l} \frac{r+f+1}{c}<x$, then $M_{T-1}=\frac{1}{2} x, L_{T-1}=0$.

- If $\frac{1}{4}<p_{l} \leq 1$, then there are three scenarios:

1. $0 \leq x \leq \frac{r+f+1}{c}$, then $M_{T-1}=0, L_{T-1}=x$;

2. $\frac{r+f+1}{c}<x \leq \frac{r+f+1}{c} \frac{\sqrt{p_{l}}}{1-\sqrt{p_{l}}}$, then $M_{T-1}=0, L_{T-1}=\frac{r+f+1+c x}{2 c}$;

3. $\frac{r+f+1}{c} \frac{\sqrt{p_{l}}}{1-\sqrt{p_{l}}}<x$, then $M_{T-1}=\frac{1}{2} x, L_{T-1}=0$.

\section{Summary and Discussions}

This paper presents some simple and stylish models for the optimal placement problem in the LOB and analyzes optimal strategies in each model. There are many possibilities to generalize the models. For instance, one could consider the possibility of an intermediate selling, which brings the optimal placement problem closer to the market making problem, where trading strategies involve simultaneously placing limit and market orders to buy and sell. The idea then is to maximize the profit by playing with the spread between the bid and ask prices, while controlling the inventory risk and the execution risk. See for example, Avelaneda and Stoikov (2008) [8], Bayraktar and Ludkovski (2012)[9], Cartea and Jaimungal (2013) [14, Cartea, Jaimugal, and Ricci (2011)[15, Veraarta (2010) [49], Guilbaud and Pham (2011) [29], Guéant, Lehalla, and Tapia (2012) [28], and Horst, Lehalle and Li (2013) [33. There are also order scheduling problems especially when orders can be placed in different exchanges with different fee structures. See Laruelle, Lehalle, and Pagès (2011) [37] and Cont and Kukanov (2012) [18].

Acknowledgement: The first author would like to thank R. Cont, S. Jaimungal, A. Kercheval, A. de Larrard, D. Leinweber, J. Ma, C. Moallemi, J. Wu, S. Wong, K. Wong, and the participants in WCMF 2012, SIAM Conference on Financial Mathematics and Engineering 2012, and IMS-FPS2013 for comments and discussions. Generous financial and data support from NASDAQ OMX Education Group, New York, and CASH Dynamic Opportunities Investment Lt., Hong Kong is acknowledged. 


\section{References}

[1] Alfonsi, A., Fruth, A. and Schied, A. (2010). Optimal execution strategies in limit order books with general shape functions. Quantitative Finance, 10(2), 143-157.

[2] Alfonsi, A., Schied, A. and Slynko, A. (2012). Order book resilience, price manipulation, and the positive portfolio problem. SIAM J. on Financial Mathematics, 3, 511-533.

[3] Allaart, P. (2004). Optimal stopping rules for correlated random walks with a discount. J. Appl. Probab., 41(2), 483-496.

[4] Almgren, R. (2003). Optimal execution with nonlinear impact function and trading enhanced risk. Applied Mathematical Finance, 10, 1-18.

[5] Almgren, R. and Chriss, N. (1999). Value under liquidation. Risk, 12, 61-63.

[6] Almgren, R. and Chriss, N. (2000). Optimal execution of portfolio transactions. Journal of Risk, $3,5-39$.

[7] Almgren, R. and Lorenz, J. (2007). Adaptive arrival price. Institutional Investor Journals, 1, 59-66.

[8] Avellaneda, M. and Stoikova, S. (2008) High-frequency trading in a limit order book. Quantitative Finance, 8(3), 217-224.

[9] Bayraktar, E. and Ludkovski, M. (2012). Liquidation in limit order books with controlled intensity. Mathematical Finance, to appear.

[10] Bertsimas, D. and Lo, A.W. (1998). Optimal control of execution codes. Journal of Financial Markets, 1, 1-50.

[11] Biais, B., Hillion, P., and Spatt, C. (1995). An empirical analysis of the limit order book and the order flow in the Paris Bourse. Journal of Finance, 50(5), 1655-1689.

[12] Bouchaud, J-P., Mezard, M., and Potters, M. (2002). Statistical properties of stock order books: Empirical results and models. Quantitative Finance, 2, 251-256.

[13] Brogaard, J., Hendershott, T., and Riordan, R. (2012). High frequency trading and price discovery. Working Paper.

[14] Cartea, A. and Jaimungal, S. (2013). Modeling asset prices for algorithmic and high frequency trading. Applied Mathematical Finance, Forthcoming.

[15] Cartea, A., Jaimungal, S., and Ricci, J. (2011). Buy low sell high: A high frequency trading perspective. Preprint. University College London.

[16] Chen, F. and Hall, P. (2013) Inference for a non-stationary self-exciting point process with an application in ultra-high frequency financial data modeling" Journal of Applied Probability, Forthcoming.

[17] Cont, R. (2011) Statistical Modeling of High Frequency Financial Data: Facts, Models and Challenges. IEEE Signal Processing, Volume 28, No 5, 16-25.

[18] Cont, R. and Kukanov, A. (2012). Optimal order placement in limit order markets. Preprint. 
[19] Cont, R., Kukanov, A., and Stoikov, S. (2013). The price impact of order book events. Jornal of Financial Econometrics, Forthcoming.

[20] Cont, R., Talreja, R., and Stoikov, S. (2010) A stochastic model for order book dynamics. Operations Research, 58(3), 549-563.

[21] Cvitanić, J. and Kirilenko, A. (2010). High-frequency traders and asset prices. Working paper.

[22] Easley, D., de Prado, L. M., and O'Hara, M. (2012). Flow toxicity and liquidity in a high-frequency world. Review of Financial Studies, 25(5), 1457-1493.

[23] Forsyth, P.A., Kennedy, J.S., Tse, S.T. and Windcliff, H. (2011). Optimal trade execution: a mean-quadratic-variation approach. Preprint.

[24] Gatheral, J. (2010). No-dynamic-arbitrage and market impact. Quantitative Finance, 10(7), 749759.

[25] Gatheral, J. and Schied, A. (2011). Optimal trade execution under geometric Brownian motion in the Almgren and Chriss framework. International Journal on Theoretical and Applied Finance, 14 (3), 353-368.

[26] Gillis, J. (1955). Correlated random walk Mathematical Proceedings of the Cambridge Philosophical Society. 51(4), 639-651.

[27] Goldstein, S. (1951). On diffusion by discontinuous movements, and on the telegraph equation. The Quarterly Journal of Mechanics and Applied Mathematics. 4(2), 129-156.

[28] Guéant, O., Lehalle, C-A., and Tapia, F. J. (2012). Optimal portfolio liquidation with limit orders. Preprint.

[29] Guilbaud, F. and Pham, H. (2013). Optimal high frequency trading with limit and market orders. Quantitative Finance, 13(1), 79-94.

[30] Guo, X., and Zervos, M. (2012). Optimal execution with nonlinear and multiplicative price impact Preprint.

[31] He, H. and Mamaysky. (2005). Dynamic trading with price impact. Journal of Economic Dynamics and Control, 29, 891-930.

[32] Hollifield, B., Miller, R. A., and Sandas, P. (2004). Empirical analysis of limit order markets. Review of Economic Studies, 71, 1024-1063.

[33] Horst, U., Lehalle, C-A. , and Li Q. H. (2013). Optimal trading in a two-sided limit order book. Preprint.

[34] Huberman, G. and Stanzl, W. (2004). Price manipulation and quasi-arbitrage. Econometrica, $72(4), 1247-1275$.

[35] Hult, H. and Kiessling, J. (2010). Algorithmic trading with Markov chains, Doctoral thesis, Stockholm University, Sweden.

[36] Kirilenko, A., Kyle A., Samadi M., and Tuzun T. (2011). The flash crash: the impact of high frequency trading on an electronic market. Preprint. 
[37] Laruelle, S., Lehalle, C-A, and Pagès, G. (2011). Optimal split of orders across liquidity pools: a stochastic algorithm approach. SIAM Journal on Financial Mathematics, 2(1), 1042-1076.

[38] Moallemi, C., Park, B. and Van Roy, B. (2009). The execution game. Working paper.

[39] Mohan, C. (1955). The gambler's ruin problem with correlation. Biometrika, 42(3/4), 486-493.

[40] Renshaw, E. and Henderson, R. (1981). The correlated random walk. Journal of Applied Probability. 403-414

[41] Redner, Sidney (2001). A guide to first-passage processes. Cambridge University Press.

[42] Obizhaeva, A. and Wang, J. (2013). Optimal trading strategy and supply/demand dynamics. Journal of Financial Markets, 16(1), 1-32.

[43] Predoiu, S., Shaikhet,G., and Shreve, S. (2011). Optimal execution in a general one-sided limit-order book. SIAM J. Finan. Math. 2, 183-212.

[44] Potters, M. and Bouchaud, J-P. (2003). More statistical properties of order books and price impact. Physica A, 3(24), 133-140.

[45] Renshaw, E. and Henderson, R. (1981). The correlated random walk. Journal of Applied Probability, 18(2), 403-414.

[46] Rosu, I. (2009) A Dynamic model of the limit order book. Review of Financial Studies 22, 46014641.

[47] Schied, I. and Schöneborn, T. (2009). Risk aversion and the dynamics of optimal liquidation strategies in illiquid markets. Finance and Stochastics, 13, 181-204.

[48] Schied, I., Schöneborn, T., and Tehranchi (2010). Optimal basket liqudition for CARA investors is deterministic. Applied Mathematical Finance, 17(6), 471-489.

[49] Veraarta, L. A. M. (2010). Optimal market making in the foreign exchange market. Applied Mathematical Finance, 17(4), 359-372.

[50] Weiss, A. (2009). Executing large orders in a microscopic market model. Available at http://arxiv.org/abs/0904.4131v1.

[51] Zheng, B., Moulines, E., and Abergel, F. (2012). Price jump prediction in limit order book. Working Paper. 